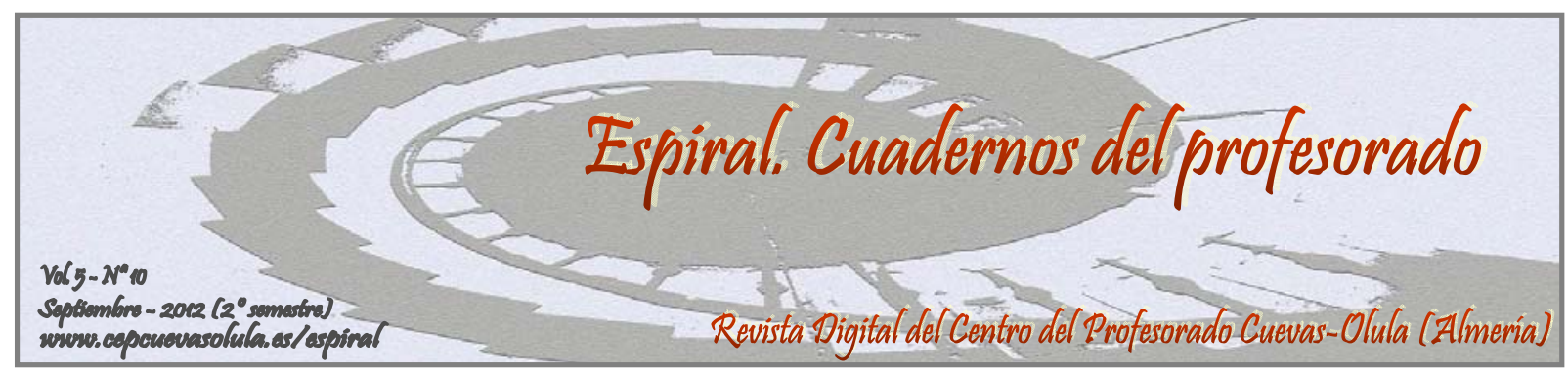

\title{
LA DISCIPLINA ESCOLAR DESDE UN ENFOQUE PSICOEDUCATIVO PARA PROMOVER UNA INTERVENCIÓN EFECTIVA DEL PROFESORADO
}

\author{
THEORY-PRACTICE ON SCHOOL DISCIPLINE FROM A \\ PSYCHOEDUCATIONAL APPROACH TO PROMOTE EFFECTIVE \\ INTERVENTION OF THE FACULTY
}

\author{
María del Mar Badía Martín ${ }^{(1)}$, Concepción Gotzens Busquets ${ }^{(2)}$ \\ y Rosalba Zamudio Villafuerte ${ }^{(3)}$
}

(1) Departamento de Psicología Básica, Evolutiva y de la Educación, Universidad Autónoma de Barcelona, España

(2) Departamento de Pedagogía Aplicada y Psicología de la Educación, Universidad de las Islas Baleares, España

(3) Instituto Superior de Ciencias de la Educación del Estado de México, México

RESUMEN: La disciplina es un asunto técnico y como tal exige un marco de acción pertinente. Pero las circunstancias bajo las cuales el profesor la gestiona, muchas veces no responde a un cuerpo teórico formal. Esto hace que no pueda sustentarse la planificación de las acciones a operarse, con la finalidad de conseguir un clima óptimo para el proceso instruccional. Al carecer de una formación en el control del aula, el profesorado día a día se enfrenta a los problemas de indisciplina con recursos poco fiables. En nuestro estudio la población participante fueron tres zonas escolares adscritas al Subsistema Educativo Estatal de la Secretaría de Educación del Estado de México. Se elaboró un cuestionario específico con escenarios instruccionales que fue aplicado a los docentes mexicanos. Los datos mostraron evidencias que las formas para establecer la disciplina en clase, no responden a las disposiciones de un campo de conocimiento formal en estos temas.

Palabras clave: disciplina escolar, formación, estrategias, conocimiento, creencias.

ABSTRACT: Discipline is a technical matter and as such requires a framework for appropriate action. But the circumstances under which the teacher manages classroom, not a theoretical answer to formal psychology perspective underpin the planning of actions to be operated with the aim of achieving an optimal climate for the instructional process. Lacking control training in the classroom, teachers face every day to discipline problems with unreliable resources. The participant population was three school zones subsystem attached to the State Education Ministry of Education of the State of Mexico. A questionnaire was developed specifically with instructional scenario was applied to the Mexican teachers. The data showed evidence that the ways to establish discipline in class, do not respond fully to the provisions of a formal field of knowledge in these subjects.

Key words: school discipline, training, strategies, knowledge, beliefs. 
Badía Martín, M.M., Gotzens Busquets, C., y Zamudio Villafuerte, R. (2012). La disciplina escolar desde un enfoque psicoeducativo para promover una intervención efectiva del profesorado. Espiral. Cuadernos del Profesorado, 5(10), 65-77. Disponible en: http://www.cepcuevasolula.es/espiral.

Fecha de recepción: 06/02/2012

Fecha de aceptación: 07/05/2012
Enviar correspondencia a: mar.badia@uab.es

\section{1.- INTRODUCCIÓN}

Teóricamente la disciplina hace referencia al conjunto de normas, reglas y procedimientos aplicados al contexto del aula, cuya finalidad es conseguir las mejores condiciones para que el proceso de enseñanza-aprendizaje llegue a sus objetivos previstos. En concreto de la disciplina derivan las orientaciones puntuales para no rebasar los medios asequibles y salvar problemas.

Si bien las situaciones de indisciplina o de acoso entre los escolares han existido siempre, éstas cobran cada vez más relevancia debido a las dificultades para poder llevar a cabo el proceso de enseñanza-aprendizaje (Veiga y Rodríguez, 2008). Evidentemente el profesorado vive problemas de comportamiento en el desarrollo de sus clases y los procedimientos para solventarlos obedecen más a la experiencia que no al conocimiento científico que tengan del tema; no es de extrañarse entonces el fracaso en la consecución de los propósitos del proceso enseñanza-aprendizaje; cuyo responsable invariablemente es el docente.

Jones y Jones, (1990) aducen que por lo general los docentes se sienten frustrados por su falta de pericia para determinar el origen del comportamiento disruptivo en el aula. Con la finalidad de conseguir un ambiente óptimo para enseñar y aprender, la disciplina escolar regula de manera precisa las interacciones del alumnado. Sin embargo su comportamiento en el aula nunca se debe considerar en forma aislada; por el contrario, ha de tenerse presente que el niño constituye el centro de una matriz de fuerzas interrelacionadas. Explicar las situaciones instruccionales como intrínsecamente interactivas, significa que nada de lo que ocurra en ellas es ajeno a ninguno de sus miembros y, consecuentemente tampoco es imputable a nadie en exclusiva; la interactividad supone todo y a todos.

En este mismo entramado tiene efecto la disrupción en el aula; entendida como un estado de inquietud y desorden en la clase con las consiguientes dificultades en el desarrollo de las tareas cotidianas del aula. Geiger (2000) cit. Calvo et al. (2005), define las conductas disruptivas como las acciones de los alumnos que distraen, perturban, compiten o amenazan, y pueden ir desde las conductas benignas a actos graves de agresión.

Las conductas calificadas como disruptivas tienen diferentes rangos de gravedad y Calvo et al. (2005) las clasifica teniendo en cuenta cuatro parámetros:

$\checkmark$ Las normas: llegar tarde o faltar a clase, deteriorar el material, desorden del mobiliario, comer en clase, indumentaria estrafalaria, realizar ruido o gritos, falta de orden a las salidas y entradas de clase son muestras representativas.

$\checkmark$ La tarea: no traer los deberes, rehusar hacer las tareas en clase, hacer comentarios vejatorios, falta de interés, pasividad e inactividad o preguntar insistentemente con ánimo de retrasar, resultan casos característicos.

$\checkmark$ Respeto al profesor: platicar cuando éste habla, no acatar sus órdenes, levantarse sin permiso de su sitio y amenazar al maestro, constituyen comportamientos frecuentes en el aula.

$\checkmark$ La relación con los compañeros: pelearse con un igual, reírse de él o insultarlo y hacer gestos jocosos forman parte del hacer casi diario de los alumnos.

Es preocupante que la convivencia en los centros se convierta a veces en buscar recetas o soluciones mágicas fuera de la propia escuela porque puede resultar incluso más cómodo y menos traumático que descubrir la cantidad de prácticas, costumbres y culturas que la institución emplea y podrían ser susceptibles de revisión. 
Las investigaciones más recientes sobre conflictividad en las aulas españolas y mexicanas reflejan una preocupación mayoritaria de los docentes por episodios de indisciplina caracterizados por su baja intensidad y elevada frecuencia. En su conjunto, desligados de acciones de violencia explícita, que se presenta muy ocasionalmente y que aunque no por ello revisten menor gravedad, constituyen un serio problema que obstaculiza la acción educativa. Es por ello que se considera importante un estudio de procedimientos que permitan prevenir la aparición de estos problemas. En la literatura consultada se han podido revisar diversos artículos acerca de las bondades de la participación comunitaria en los centros escolares, pero no dejan de haber limitaciones ya que mayoritariamente una parte importante de estos casos son de participación testimonial y esporádica, cosa que no facilita una labor e implicación directa en los centros.

Además, por lo general el profesorado desatiende un marco científico para establecer la disciplina en el aula, y los niveles de atención puestos en los problemas de indisciplina no son del todo deseables. Un estudio realizado por Estrela (2005), califica las intervenciones disciplinarias de los maestros como participaciones fundadas sobre todo en su intuición y experiencia, más que en la aplicación sistemática de una teoría psicológica o pedagógica determinada. De ese modo, esas intervenciones en general resultan relativamente limitadas.

Hablar de disciplina es hablar de procesos de enseñanza-aprendizaje y por consiguiente:

- Es aprendizaje por parte del alumnado.

- Es un instrumento cuya finalidad primera es garantizar el orden suficiente en el grupo para facilitar si funcionamiento y derivadamente, solucionar problemas en caso de presentarse.

- Es un medio para conseguir el éxito instruccional.

- La disciplina escolar no constituye un recetario de propuestas con las cuales enfrentar problemas de comportamiento. Es un enfoque global de organización y dinámica del comportamiento en la escuela y en el aula.

- Las orientaciones emanadas de la disciplina escolar son coherente con los propósitos instruccionales, lo que garantiza la solución de problemas concretos propios del aula, asimismo previene la aparición de éstos.

- La disciplina no es sinónimo de represión, sino de aprendizaje y socialización.

Este artículo argumenta las formas de asesorar a los docentes de educación primaria a propuestas factibles para la gestión de la disciplina escolar. Demuestra asimismo cuáles son las fallas en el conocimiento puntual en torno a los temas de disciplina escolar, seguido de los medios para salvar esta situación después de haber trabajado sobre los siguientes planteamientos: ¿Cuáles son los conocimientos del profesor sobre los temas de disciplina escolar?, ¿Bajo qué percepciones la establece en el aula?, ¿Cuál es la gama de conocimientos que el profesorado debería integrar a sus acciones en el aula, cuando éstas tienen relación con la disciplina escolar? y, ¿Qué factores posibilitan superar la barrera de las creencias para asumir actitudes profesionales en beneficio de la gestión de la disciplina escolar?

\section{2.- MATERIAL Y MÉTODO}

Para poner a prueba las categorías en estudio, la conducción del trabajo empírico se llevó a cabo desde las directrices del método hipotético-deductivo operando un diseño cuasi-experimental intrasujetos. Por ser el diseño el medio para confrontar los datos, se tuvo rigor en los siguientes factores extrínsecos:

- Control de variables extrañas durante las observaciones y el desarrollo del programa de formación, asignando para tal efecto un espacio de trabajo para los profesores participantes.

- La disposición de un aula con los materiales necesarios durante la operación del programa.

- Vigilar en los participantes el cumplimiento de los requisitos anotados previamente: ser profesor de educación primaria y estar en contacto con un grupo. 
Asimismo se cuidaron puntualmente las etapas de operación del diseño. El pretest se verificó administrando el cuestionario con los escenarios (Verónica, Begoña y Luís). Terminada esta fase se operó el programa de formación para después aplicar la segunda parte de la encuesta considerando los escenarios (Víctor, Pablo y Elena). Se determinaron tres zonas en la muestra en estudio -A, B, C- con un tiempo específico para el desarrollo del programa con los contenidos fundamentales concernientes a la disciplina escolar; en la tabla 1 , se muestra el cronograma.

Para alcanzar la objetividad exigida en la investigación, se utilizaron los paquetes estadísticos de SPSS, en su versión 15 para Windows. A través de frecuencias, exploración, descriptivos, tablas de contingencia, resúmenes y gráficos, se explica detalladamente la información recopilada de los diferentes escenarios en sus respectivas tomas de medida, (pretest-postest). El cierre del análisis con el sustento de la prueba T-Student (tabla 1), presenta un bloque de resultados tanto a nivel general como individual, cuya información fortalece las argumentaciones desprendidas de las tablas con el concentrado de los datos globales.

Tabla 1. Prueba de muestras relacionadas

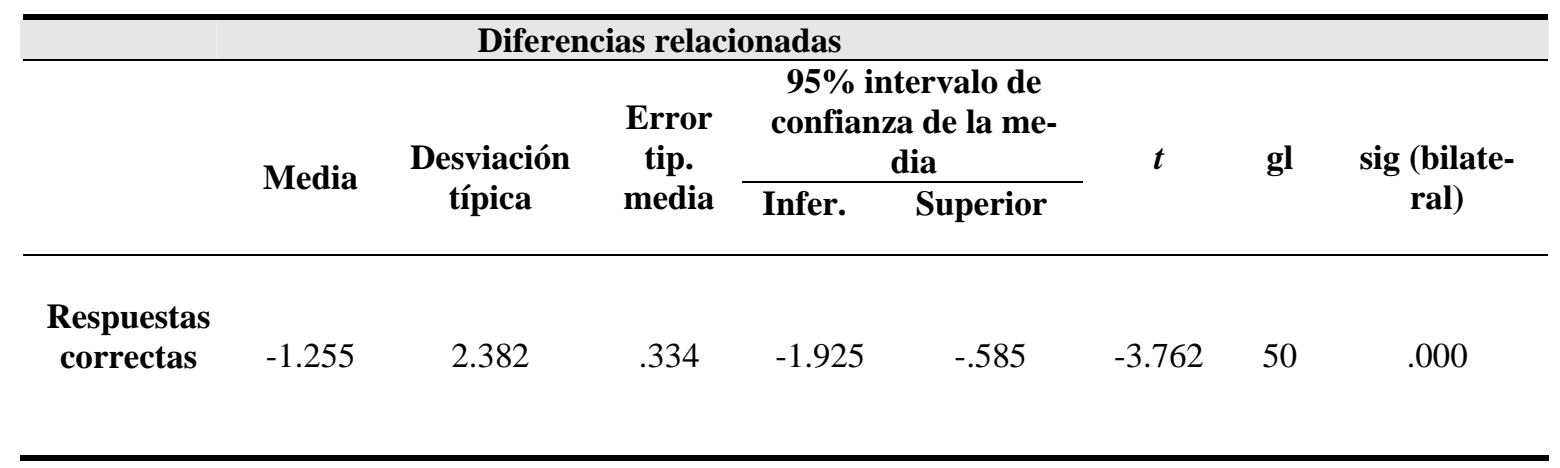

Tabla 2. Distribución del profesorado para la operación del diseño

\begin{tabular}{ccccc}
\hline Zona & Sede de trabajo & Distribución horaria & $\begin{array}{c}\text { Profesores } \\
\text { asistentes }\end{array}$ & $\begin{array}{c}\text { Profesores } \\
\text { colaboradores }\end{array}$ \\
\hline “A” & $\begin{array}{c}\text { Escuela Primaria “Mario } \\
\text { Colín Sánchez” }\end{array}$ & $\begin{array}{c}\text { Sesiones sabatinas de } \\
8: 00 \text { a 14:00 hrs. }\end{array}$ & 25 & 20 \\
“B” & $\begin{array}{c}\text { Escuela Primaria “Lázaro } \\
\text { Cárdenas del Río” }\end{array}$ & $\begin{array}{c}\text { Sesiones vespertinas de } \\
14: 00 \text { a 19:00 hrs. }\end{array}$ & 22 & 15 \\
“C” & $\begin{array}{c}\text { Escuela Primaria “Adolfo } \\
\text { López Mateos” }\end{array}$ & $\begin{array}{c}\text { Sesiones vespertinas de } \\
13: 30 \text { a 18:30 hrs. }\end{array}$ & 23 & 16 \\
\hline
\end{tabular}

\section{Muestra}

En una población de 504 profesores de educación primaria, participaron tres zonas escolares P027 P043 y P046 adscritas al Subsistema Educativo Estatal de la Secretaría de Educación del Estado de México, específicamente en los Municipios de Ecatepec de Morelos y Chicoloapan de Juárez.

Con el respaldo de un procedimiento no aleatorio se logró integrar a 51 profesores como muestra. La opción de un muestreo no probabilístico buscó una participación voluntaria, puesto que el desarrollo del programa se llevó a cabo en horarios distintos a su labor en las aulas. De la muestra de 51 profesores; un $94.1 \%$ correspondieron al sexo femenino y $5.9 \%$ masculino.

\section{Instrumentos}

Conscientes de lo espinoso que resulta tratar tópicos de disciplina escolar, y prestos ante la dificultad de obtener información fidedigna relacionada con el pensamiento de los docentes, se recurrió a un instrumento fiable para tal efecto propuesto en Zamudio (2009). El cuestionario contiene 15 ítems a contestar a partir de la descripción de escenarios por cada grado de educación primaria. Primero, 
tercero y sexto fueron empleados de prestest, segundo cuarto y sexto como postest. En el espacio instaurado entre ambos momentos se desarrolló el programa de formación bajo el título "El enfoque preventivo de la disciplina escolar, una aplicación psicoeducativa en el aula", sustentado en el modelo de entrenamiento de la propuesta de Imbernón (1998).

Algunos de los módulos y contenidos del programa de formación estaban relacionados con: precisiones conceptuales sobre la disciplina escolar, la percepción de la disciplina en el aula, el enfoque preventivo de la disciplina escolar, intervención del docente ante los problemas de comportamiento, etc.

\section{Procedimiento}

Con el cuestionario como instrumento de trabajo de campo se llevó a cabo una recopilación inicial de datos, consecutivamente la operación del programa de formación y finalmente, una nueva medición con el mismo instrumento. Previo al desarrollo del programa, se consiguió la colaboración del personal directivo en tres zonas escolares de educación primaria de las cuales se sustrajo la muestra.

Con el apoyo de un dossier los contenidos temáticos alrededor de disciplina escolar se abordaron en jornadas de trabajo promoviendo en el profesorado el análisis y la reflexión a partir de un contexto titulado “Qué pasa en la escuela de Óscar?” con cuatro escenarios -distintos a los planteados en el cuestionario- como referente:

- $\quad$ Primer grado (Profesora Diana).

- Segundo grado (Profesora Alejandra).

- Tercer grado (Profesor Arturo).

- Cuarto grado (Profesora Maritza).

\section{Análisis estadístico}

El análisis estadístico de los datos se efectuó mediante los paquetes estadísticos de SPSS, en su versión 15 para Windows a través de frecuencias, exploración, descriptivos, tablas de contingencia, resúmenes y gráficos para cada una de las categorías en estudio referidas en la tabla 2.

Tabla 3. Las categorías de estudio en el instrumento

\begin{tabular}{|c|c|}
\hline Categoría de estudio & Preguntas incluidas en el cuestionario \\
\hline & ${ }^{*}$ ¿En este grupo hay normas de disciplina? \\
\hline Normas de disciplina & $\begin{array}{l}\text { *CCuál o cuáles son los enunciados que definen correctamente la disciplina en } \\
\text { este grupo? }\end{array}$ \\
\hline $\begin{array}{l}\text { Comunicación de } \\
\text { normas }\end{array}$ & * ¿Estima que el profesor ha indicado a los alumnos la existencia de normas? \\
\hline $\begin{array}{l}\text { Entendimiento de } \\
\text { normas }\end{array}$ & * ¿Las normas a cumplir por los alumnos están claras y precisas? \\
\hline $\begin{array}{l}\text { Organización- } \\
\text { disciplina en clase }\end{array}$ & * ¿Cómo valora las características de la organización de la clase del profesor? \\
\hline $\begin{array}{l}\text { Participación del } \\
\text { profesor }\end{array}$ & $\begin{array}{l}\text { *El momento de la intervención del profesor ante las conductas que alteraron el } \\
\text { orden de la disciplina fue: }\end{array}$ \\
\hline $\begin{array}{l}\text { Orientación de la } \\
\text { clase }\end{array}$ & $\begin{array}{l}\text { *Las instrucciones proporcionadas por el profesor para la realización de tareas } \\
\text { por parte del grupo, se caracteriza por: }\end{array}$ \\
\hline $\begin{array}{l}\text { Prevención de la } \\
\text { disciplina }\end{array}$ & $\begin{array}{l}\text { *Si el profesor tiene la intención de prevenir conductas disruptivas } \\
\text { (perturbadoras) durante el proceso instruccional de este grupo, ¿Cuál es la } \\
\text { estrategia más apremiante? }\end{array}$ \\
\hline
\end{tabular}




\begin{tabular}{|c|c|}
\hline $\begin{array}{l}\text { Conductas disruptivas } \\
\text { del } \\
\text { alumnado }\end{array}$ & 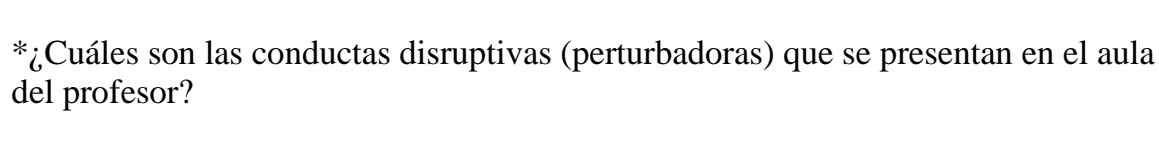 \\
\hline $\begin{array}{l}\text { Desarrollo del } \\
\text { aprendizaje }\end{array}$ & ${ }^{*}$ ¿Cómo influyeron en el aprendizaje las conductas disruptivas presentadas? \\
\hline $\begin{array}{l}\text { Alcances de la } \\
\text { intervención docente } \\
\text { en la disciplina }\end{array}$ & $\begin{array}{l}\text { * ¿Cómo explica la participación del profesor (a) en torno a la gestión de la } \\
\text { disciplina en clase? }\end{array}$ \\
\hline $\begin{array}{l}* \text { Perfil de la } \\
\text { participación docente } \\
\text { en la disciplina }\end{array}$ & $\begin{array}{l}\text { * Las medidas disciplinarias puestas en práctica por el profesor durante el } \\
\text { desarrollo de la clase las considera: }\end{array}$ \\
\hline $\begin{array}{l}\text { * Calidad de las } \\
\text { medidas disciplinarias }\end{array}$ & $\begin{array}{l}\text { *De las acciones emprendidas por el profesor, seleccione y subraye las más } \\
\text { efectivas para la disciplina de este grupo. }\end{array}$ \\
\hline $\begin{array}{l}\text { *Relación de } \\
\text { estrategias efectivas } \\
\text { para la disciplina }\end{array}$ & $\begin{array}{l}\text { * ¿Qué recomendaciones usted proporciona al profesor para establecer y/o mejorar } \\
\text { la disciplina? Elija las sugerencias, ordenándolas de mayor a menor importancia } \\
\text { (1 para la de mayor importancia, seguida de } 2 \text {, y así sucesivamente, hasta llegar a } \\
\text { la menos recomendable). }\end{array}$ \\
\hline $\begin{array}{l}\text { *Escala de estrategias } \\
\text { para establecer la } \\
\text { disciplina en el aula }\end{array}$ & $\begin{array}{l}\text { * En relación a las medidas disciplinarias emprendidas por el* profesor, marque } \\
\text { su punto de vista con una }(*) \text { su acuerdo o desacuerdo en el casillero } \\
\text { correspondiente. }\end{array}$ \\
\hline
\end{tabular}

\section{3.- RESULTADOS Y DISCUSIÓN}

La preparación del profesorado para crear y mantener un ambiente óptimo de aprendizaje, exige conocimientos y habilidades que les permitan gestionar una organización efectiva de la clase, estableciendo reglas y procedimientos, desarrollando relaciones con los niños, mantener la atención del alumnado y motivarlos hacia las actividades académicas Lepage et al. (2005), pero ¿Qué saben los profesores sobre disciplina escolar? ¿Cómo la establecen en el aula? ¿Qué se debe saber al respecto? ¿Qué consigue disciplina en el proceso instruccional?

La investigación realizada encontró respuesta a los planteamientos anteriores, estado del conocimiento con importantes alcances en el proceso enseñanza-aprendizaje. Se partió de determinar el nivel de acierto y error de las respuestas del profesorado en cada fase de la administración del cuestionario; revisión efectuada sobre la base de un concentrado de respuestas esperadas para cada una de las categorías de estudio.

La mejor trayectoria de las respuestas del profesorado se manifestó en la categoría referente a la organización de la disciplina, consiguiendo un $82.4 \%$ de acierto sobre el antecedente de $31.4 \%$ en el pretest, mejora que por supuesto involucra conocimientos, actitudes y habilidades psicoeducativas para gestionar la disciplina en el aula de manera efectiva. "La clave del control eficaz de la clase en las diferentes edades radica en la comprensión de qué es lo que realmente desencadena el comportamiento de los niños y en la comprensión de las estrategias más apropiadas para guiarlos y orientarlos” (Fontana, 1994, p. 25).

Conseguir efectividad en el control de la clase exige al maestro conocimientos teóricos precisos relativos a la disciplina escolar. En tanto que guía, facilitador y responsable de la situación instruccional, las decisiones del docente han de favorecer la atmósfera donde se verifica este proceso.

Los resultados coinciden con Calvo et al. (2005) cuando consideran imprescindibles los siguientes parámetros: cumplir las normas, realizar la tarea, respeto al profesor, etc., para una mayor efectividad instruccional. También coinciden con Veiga y Rodríguez, (2008) cuando comentan las dificultades con las que se encuentran los docentes para poder llevar a cabo el proceso de enseñanzaaprendizaje, debido a una planificación poco rigurosa de la disciplina escolar. 
De acuerdo con los datos mostrados en las tablas 3 y 4, se verificó un avance bastante congruente en cuanto a las explicaciones de la organización-disciplina en el aula; puesto que los maestros establecieron una relación entre disciplina y organización del proceso enseñanza-aprendizaje.

Tabla 4. Organización-disciplina, pretest

\begin{tabular}{ccccccc}
\hline & \multicolumn{2}{c}{ Escenario/Verónica } & \multicolumn{2}{c}{ Escenario/Begoña } & \multicolumn{2}{c}{ Escenario/Luís } \\
\cline { 2 - 7 } & Frecuencia & $\mathbf{\%}$ & Frecuencia & $\mathbf{\%}$ & Frecuencia & $\%$ \\
\hline $\begin{array}{c}\text { Nota } \\
\text { aceptable }\end{array}$ & 44 & 86.2 & 18 & 35.3 & 10 & 19.6 \\
$\quad$ Nota \\
cercana
\end{tabular}

Tabla 5. Organización-disciplina, postest

\begin{tabular}{ccccccc}
\hline & \multicolumn{2}{c}{ Escenario/Pablo } & \multicolumn{2}{c}{ Escenario/Víctor } & \multicolumn{2}{c}{ Escenario/Elena } \\
\cline { 2 - 7 } & Frecuencia & \% & Frecuencia & \% & Frecuencia & \% \\
\hline $\begin{array}{c}\text { Nota } \\
\text { aceptable }\end{array}$ & 36 & 70.6 & 36 & 70.6 & 42 & 82.4 \\
$\quad$ Nota & 13 & 25.5 & 14 & 27.5 & 7 & 13.7 \\
$\begin{array}{c}\text { cercana } \\
\text { Nota }\end{array}$ & 1 & 2.0 & 1 & 2.0 & 2 & 3.9 \\
$\begin{array}{c}\text { apartada } \\
\text { Nota }\end{array}$ & 1 & 2.0 & 0 & 0 & 0 & 0 \\
$\begin{array}{c}\text { inaceptable } \\
\text { Total }\end{array}$ & 51 & 100 & 51 & 100 & 51 & 100 \\
\hline
\end{tabular}

En la figura 1 se muestra con claridad el avance en el rubro de la organización-disciplina; se ganaron notas aceptables, concretamente, los maestros reconocieron la injerencia de la disciplina en el aula. En tanto que guía, facilitador y responsable de la situación instruccional, el docente con estos elementos teóricos podrá tomar decisiones con mayor efectividad.

Una orientación preventiva surgida de la perspectiva psicoeducativa hace énfasis en la necesidad de cumplir de forma creativa con un trabajo de diseño sin perder de vista la premisa: la disciplina sirve al aprendizaje, en donde la prevención no se refiere sólo a las decisiones que toman los educadores de forma premeditada y previa a la instrucción, sino todo cuanto redunde en prever alteraciones, desde la opción de modificar una norma que de forma puntual presente más inconvenientes que ventajas para el grupo, hasta la elección del momento en que conviene recordar la necesidad de cumplirla.

En este talante una categoría con evidentes mejoras se hizo ostensible justamente en la dimensión preventiva de la disciplina; el pretest sólo captó dos encuestados optando por recomendaciones puntuales para prevenir la disciplina en el grupo, mientras que en el postest, incidieron 26 respuestas exactas, por ende el profesor desarrolló actitudes que le apoyarán a minimizar la disrupción en clase y optimizar el tiempo de aprendizaje de los alumnos.

Para tomar las decisiones adecuadas en esta previsión, el contar con una disciplina planificada, con toda seguridad brinda mayor efectividad, porque la intervención del profesor tiene el respaldo en un plan diseñado para decidir sobre las medidas a operar ante posibles incidencias, a diferencia de una intervención mediatizada por la improvisación y con efecto de remedio circunstancial; la precisión 
ganada en las respuestas de profesorado son indicios de una concienciación alrededor de la disciplina escolar (figura 2).

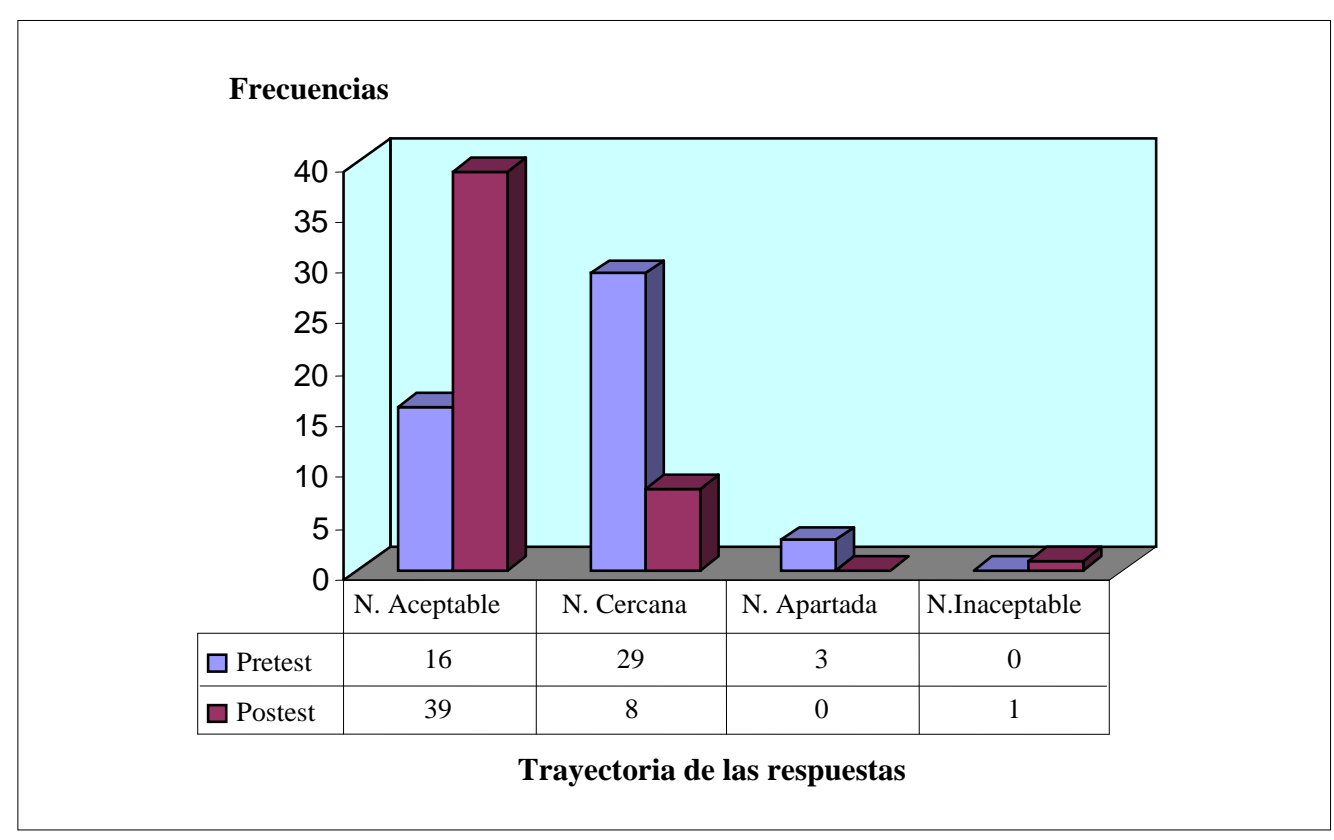

Figura 1. Organización de la disciplina

Conocimiento puntual manifestado cuando el profesorado logró diferenciar la enseñanza de valores con respecto a la disciplina escolar, por ende, se alcanzó un cambio conceptual y un rompimiento con las creencias que impiden su participación efectiva. En efecto, la disciplina tiene un carácter inmediato que permite dar a conocer las reglas a cumplir para alcanzar los fines propuestos, en tanto el aprendizaje socio-moral involucra cuestiones relacionadas con los procesos de maduración del sujeto.

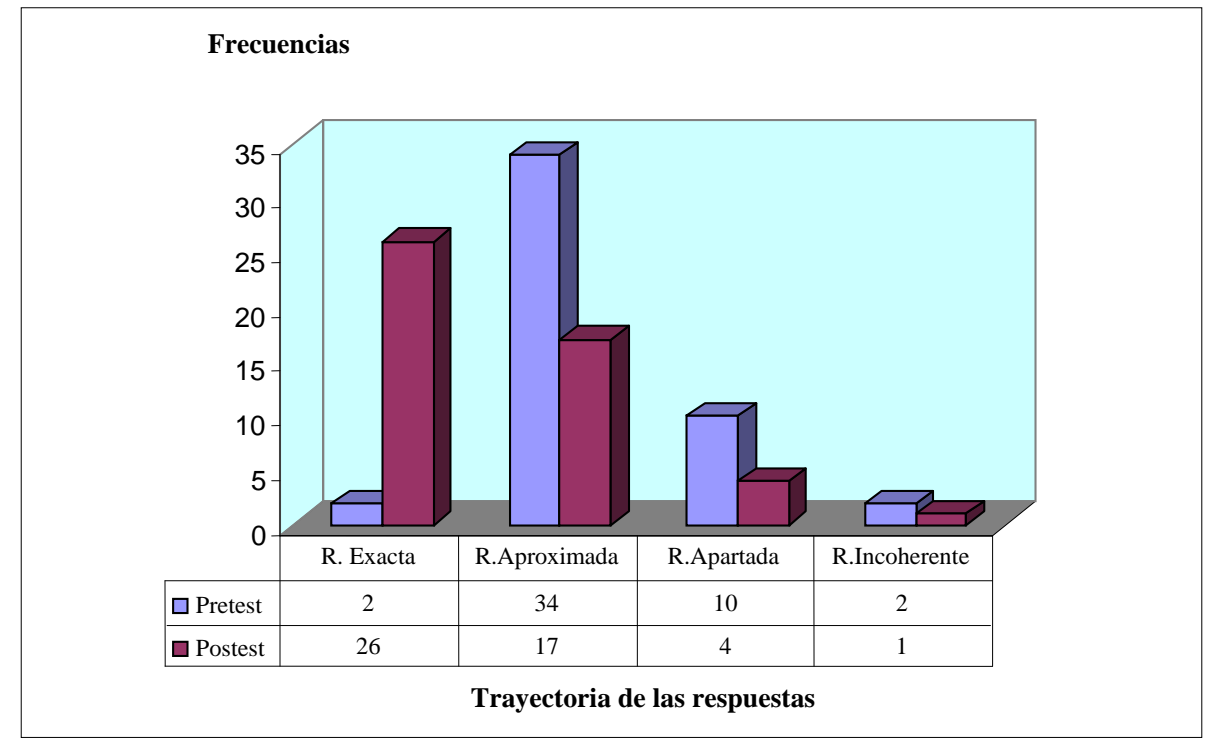

Figura 2. Prevención de la disciplina en clase

La enseñanza demanda en el profesorado conocimientos y habilidades para tomar decisiones que faciliten el proceso de llevar a cabo una clase organizada y efectiva. Una dimensión donde 
conjunta conocimientos y habilidades tiene lugar cuando el maestro efectúa un examen práctico de su desempeño en el aula, análisis que le permite reflexionar sobre su propia práctica.

Este ejercicio epistémico con toda seguridad ha de conducirlo a la comprensión de las conductas disruptivas del alumnado, pero también a reflexionar en torno a sus aciertos y errores de su proceder como promotor del aprendizaje y responsable de la convivencia en grupo, obligándolo a emprender acciones con fines de mejora en el control de clase.

La figura 3 ilustra la precisión sobre el tiempo de intervención ante conductas disruptivas, el pretest registró 22 casos correctos, en tanto el postest alcanzó los 34; así también, disminuyeron los niveles de equivocación de 19 a 7.

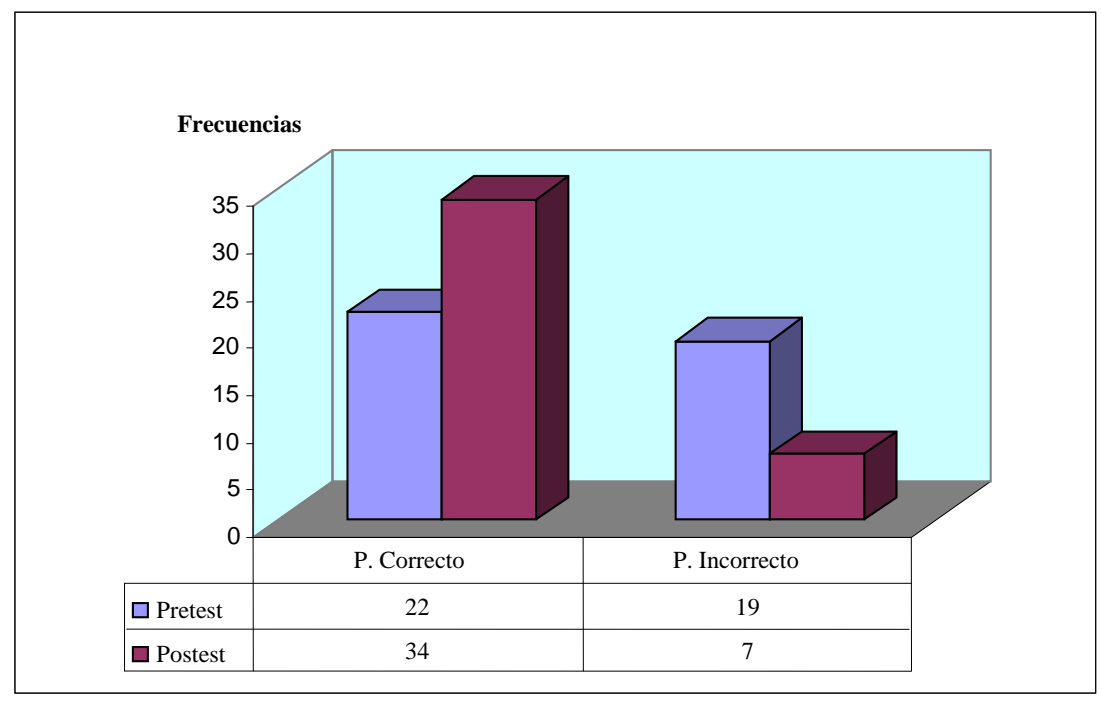

Figura 3. Tiempo de intervención ante las conductas disruptivas

El profesorado tiene un papel decisivo en la gestión de la disciplina escolar, desempeño evaluado precisamente cuando planifica y opera las estrategias para favorecerla en el aula. Enclavados ya en este espinoso tema, el momento es oportuno de dar a conocer los acuerdos y desacuerdos sobre medidas disciplinarias a la vista de las aportaciones de la psicología.

Dentro del abanico de estrategias que se nos ofertan, unas consisten en la aplicación de censuras o castigos, otras aspiran a sustituir la conducta disruptiva por otras más acordes con las normas de clase, y otras pretenden convencer al alumno de la necesidad de cambiar mediante el diálogo y la reflexión sobre el problema; e incluso otras se dirigen a involucrar a distintos miembros de la comunidad educativa con la finalidad de crear un entorno (Calvo, et al, 2005, p. 199).

Las primeras medidas corresponden a las técnicas punitivas, éstas consisten por una parte en proporcionar al alumnado una experiencia o estímulo aversivo y desagradable asociado a su mal comportamiento y por otro, privarle de experiencias o estímulo placenteros y apetecibles de manera contingente a su infracción. No resulta sencillo hallar formas de aplicación de este tipo de castigo, reduciéndose prácticamente a la aplicación de estímulos expresivos verbales (críticas y reprimendas) y no verbales ( miradas de rechazo o de desilusión); no obstante, a pesar de ello se aprecia una elevada frecuencia de uso de esta clase de castigos, probablemente por diversos motivos: por la economía de recursos que significa (bastan palabras, miradas o gestos); porque permite una aplicación inmediata y acostumbra a dar buenos resultados.

Recurrir a las estrategias punitivas en el contexto escolar no es tarea sencilla y lo es menos cuanto más se ajusta a los parámetros de trato digno y respetuoso que todo ser humano merece y de coherencia con los propósitos que enmarcan el quehacer instruccional escolar.

En este tenor, la encuesta evaluó los acuerdos y desacuerdos de los maestros en relación a medidas disciplinarias ante las incidencias de disrupción. En el pretest sobresalió la negativa para privar del recreo al alumnado (90.2\%), del mismo modo el rechazo al tiempo fuera (64.7\%) como 
medida para inhibir malos comportamientos; en cambio, acordaron involucrar al grupo para solventar las incidencias de clase (78.4\%). Fue evidente el desconocimiento del profesorado en relación los preceptos que hacen de las estrategias punitivas un recurso permisible ante los malos comportamientos. De la Mora (2003) al respecto señala que la disciplina tiene un objetivo inmediato y éste consiste en evitar o interrumpir todos aquellos comportamientos inadecuados para los procesos de aprendizaje, y la mayoría de los métodos, incluyendo el castigo tienen éxito. Afortunadamente, los docentes mostraron adelantos en este rubro, la incorporación de recursos para el manejo y control del grupo así lo confirma. Por ejemplo, acordaron suprimir el tiempo de recreo y recurrir al tiempo fuera (tabla 6).

Tabla 6. Medidas disciplinarias y punto de vista del profesorado

\begin{tabular}{|c|c|c|c|c|c|c|c|c|}
\hline \multirow{3}{*}{$\begin{array}{c}\text { Alternativas } \\
\text { Postest } \\
\end{array}$} & \multicolumn{8}{|c|}{ Estadísticos } \\
\hline & \multicolumn{2}{|c|}{ Desacuerdo } & \multicolumn{2}{|c|}{ Acuerdo } & \multicolumn{2}{|c|}{ Abstenciones } & \multicolumn{2}{|c|}{ Totales } \\
\hline & $\mathrm{F}$ & $\%$ & $\mathrm{~F}$ & $\%$ & $\mathrm{~F}$ & $\%$ & $\mathrm{~N}$ & $\%$ \\
\hline Suprimir horario de recreo & 3 & 5.9 & 43 & 84.6 & 5 & 9.6 & 51 & 100 \\
\hline Tiempo fuera & 14 & 27.5 & 31 & 60.8 & 6 & 11.8 & 51 & 100 \\
\hline Sancionar todo mal & 3 & 5.9 & 42 & 82.4 & 6 & 11.8 & 51 & 100 \\
\hline comportamiento & 22 & 43.1 & 23 & 45.1 & 6 & 11.8 & 51 & 100 \\
\hline Intervención del grupo & 0 & 0.0 & 47 & 92.2 & 4 & 7.8 & 51 & 100 \\
\hline Decisiones firmes & 0 & 0.0 & 48 & 94.1 & 3 & 5.9 & 51 & 100 \\
\hline Revisión organizada de tareas & 22 & 43.1. & 23 & 45.1 & 6 & 11.8 & 51 & 100 \\
\hline $\begin{array}{l}\text { Elevar el volumen de voz } \\
\text { Enfadarse }\end{array}$ & 13 & 25.5 & 34 & 66.7 & 4 & 7.8 & 51 & 100 \\
\hline
\end{tabular}

Para confirmar los progresos del profesorado tanto en la dimensión cognitiva como actitudinal, se presentan las medidas disciplinarias más importantes desde su punto de vista. La elaboración de normas como primera acción apareció en ambos momentos de la encuesta, aunque con más precisión en el postest; asimismo, el cumplimiento de éstas ocupó un mejor rango en la segunda fase de la encuesta. En el examen sobre la disrupción en clase en el postest, el profesorado logró una mejor identificación de los malos comportamientos. Al determinar con mayor precisión las conductas concernientes a su labor como responsable del proceso instruccional, el profesorado denotó mayor habilidad en el postest, lo cual representa un progreso meritorio en beneficio de la gestión de la disciplina del aula y del docente en su papel como autoridad educadora.

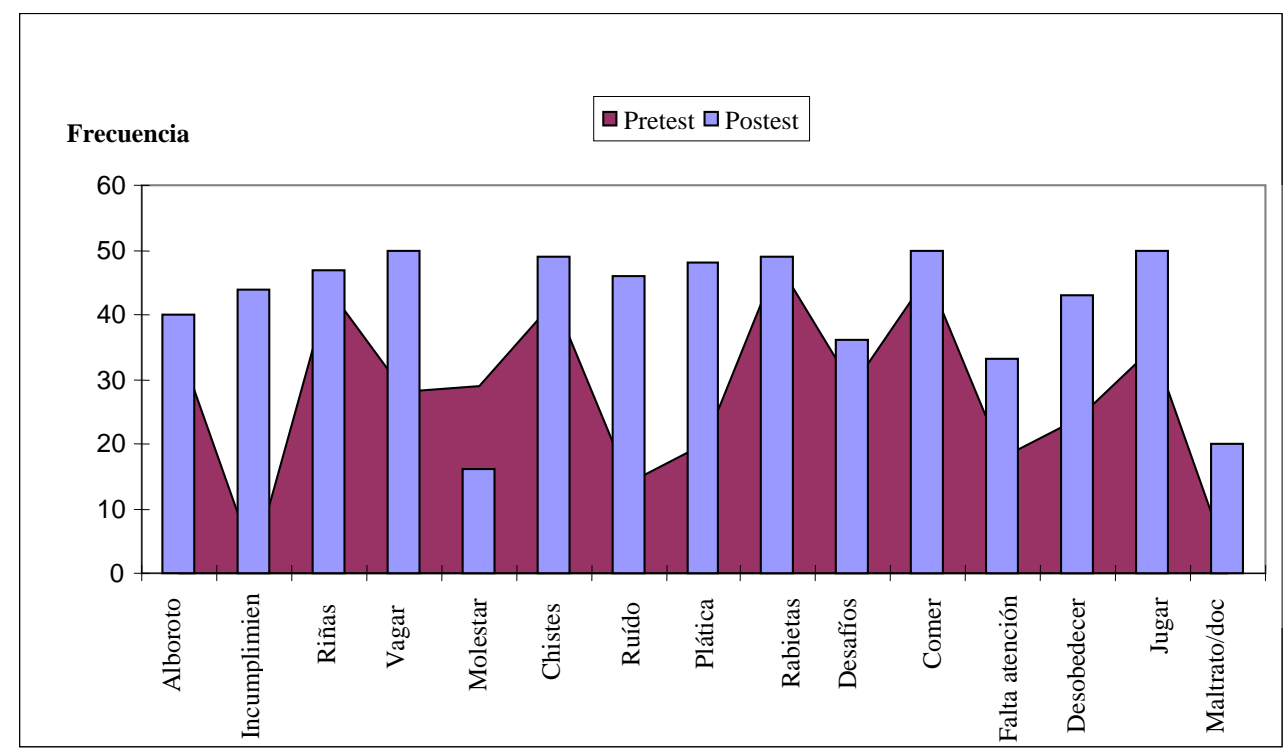

Figura 4. Precisión de respuestas a las conductas disruptivas 
En la figura 4 puede distinguirse el mayor acierto de las respuestas del profesorado como consecuencia del programa de intervención en este rubro. Las barras azules con excepción de la denominada "Molestar a quienes realizan su trabajo" superaron los logros de la primera medida. Basándonos en esta información podemos afirmar que el profesorado adquirió elementos cognitivos para determinar cuando las conductas disruptivas se presentan o no en el aula.

Los avances del profesorado para explicar la disciplina teniendo como base sus perspectivas teóricas fueron leves. En la primera fase de la encuesta, el enfoque relativo a la necesidad de enseñar a los alumnos juicios morales fue la definición más elegida al contabilizar una frecuencia de 29; en la segunda etapa, 42 profesores comprendieron tal perspectiva no es la apropiada para definir a la disciplina, y por lo tanto la excluyen.

Mantener el orden en una clase resulta ser una labor evidentemente compleja, pero no puede evadirse ya que la disciplina es un instrumento básico para el aprendizaje, y en esta perspectiva han de buscarse los procedimientos idóneos para establecerla como condición previa para el desarrollo de mencionado proceso. Sin embargo, De la Mora (2003) ha puesto de manifiesto uno de los errores más comunes de los maestros en relación al control del aula: su improvisación al enfrentar problemas de tipo conductual en el aula.

En este sentido, se averiguó la calidad de ciertas medidas disciplinarias aplicadas por el profesorado. Indiscutiblemente, se encontraron las dificultades que el profesor enfrenta para al seleccionar medidas disciplinarias que atienda en el caso del castigo los planteamientos señalados por Gotzens (1997).

- Advertido y previsible

- Inmediato:

- Significar una experiencia claramente indeseable para el alumno.

- De aplicación consistente

- Acompañarse de pautas sobre como actuar.

El programa de formación no logró generar cambios favorables para este aspecto, es decir, prevalecieron carencias en la mayoría de los maestros que les impidieron calificar adecuadamente algunas medidas disciplinarias. De igual forma, las explicaciones expuestas por el profesorado al regir sus acciones en la disciplina del aula, al tiempo de dirigir el proceso enseñanza-aprendizaje, los puntajes de aciertos en ambos momentos de la encuesta son bajos, pero no pueden ignorarse los rangos inferiores aún después del programa, situación reveladora de la dificultad del maestro para trabajar en clase atendiendo la disciplina como un requisito fundamental en el ambiente para que el proceso instruccional tenga éxito.

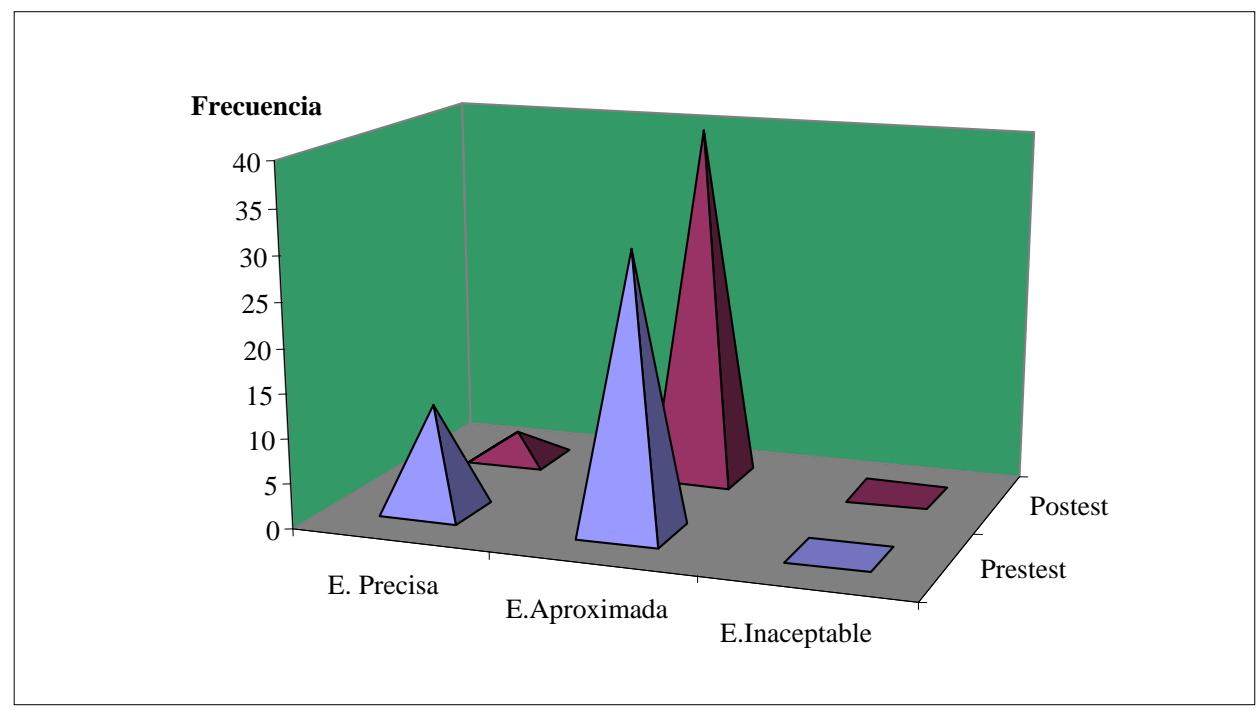

Figura 5. Perfil de la participación docente en la disciplina 
Comparando las fases de estudio, resaltó el carácter aproximado de las respuestas. Por otra parte, no se registró ningún resultado inaceptable, incluso en el postest destacan las respuestas cercanas a la cuestión planteada, (figura 5), por lo tanto las dudas persistieron aunque no debe soslayarse que un proceso de elaboración cognitiva para incorporar los contenidos estudiados a nuevas situaciones lleva su tiempo.

Una estructura y organización eficiente de la clase es imprescindible para mantener un ambiente ordenado de aprendizaje. Después de haber incorporado a los docentes al programa de formación citado, cabe ahora la pregunta ¿Mostró el profesorado indicadores teórico-prácticos que le permitan como responsable del proceso instruccional gestionar la disciplina en el aula con base al enfoque psicoeducativo? Una vez analizadas las preguntas de la encuesta conforme a las categorías en estudio, se tienen elementos para afirmar que en efecto, se dio una mejora en los conocimientos y habilidades de los maestros en temas de disciplina escolar; la figura 6 ilustra el progreso alcanzado.

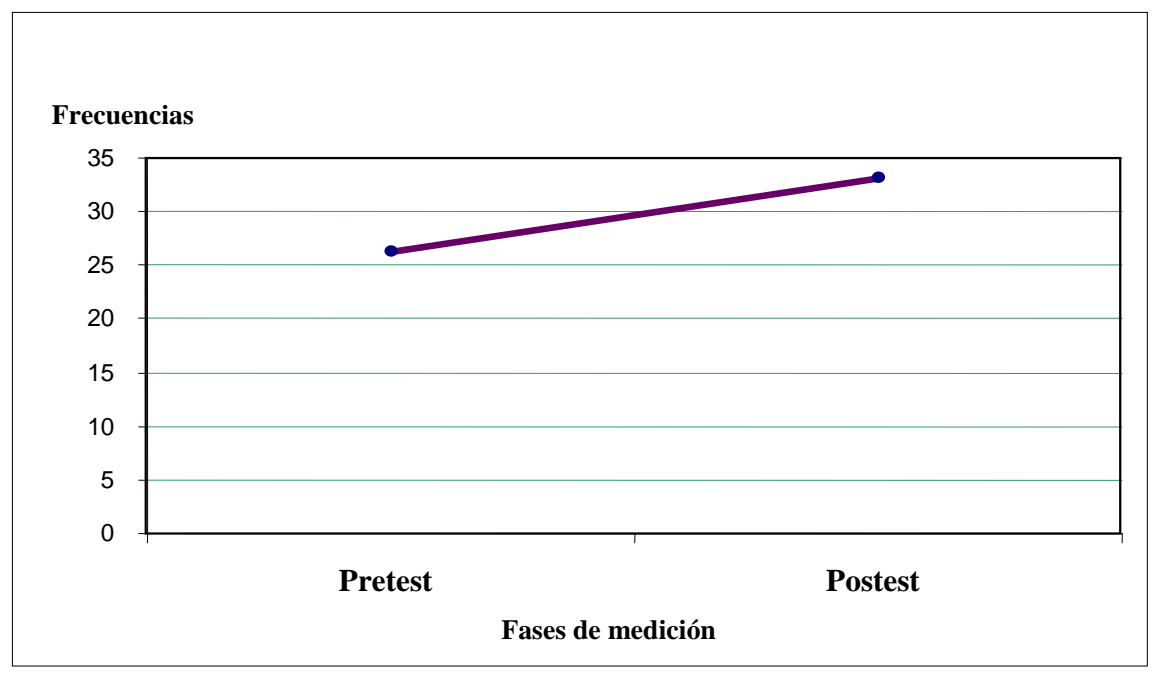

Figura 6. Impacto del programa de formación en el profesorado

Mas como se ha mostrado a lo largo de estas páginas, las mejoras no fueron homogéneas en el abordaje de los diversos temas sobre disciplina escolar, hubo avances pero también imprecisiones. Se lograron explicar cuestiones de trascendencia para la gestión de la disciplina, pero también surgieron dudas por parte de investigadores y docentes sobre si las creencias y las percepciones que se tienen sobre este tema, pudieran llegar a tener tanto peso en la población que no dejaran demasiado margen de maniobra.

\section{4.- CONCLUSIONES}

La disciplina escolar se constituye como un instrumento indispensable en el proceso instruccional, y por lo tanto, de manejo preciso por los maestros. Los datos levantados mostraron evidencias que las formas para establecer la disciplina en clase no responden a las disposiciones de un campo de conocimiento formal en estos temas; tanto a nivel teórico como práctico hay decisiones e iniciativas equivocadas del profesorado.

Una estructura y organización eficiente de la clase es imprescindible para mantener un ambiente ordenado de aprendizaje (LePage, et al., 2005). En la perspectiva psicoeducativa hay un marco viable de posibilidades para prevenir problemas de comportamiento, conseguir un ambiente de orden en el aula y optimizar el proceso enseñanza-aprendizaje, pero se demanda un profesorado consciente con respecto a los factores y elementos cuya presencia en el aula quebrantan significativamente el orden de la clase y una vez detectados, estar en posibilidades de atenderlos con base en un conjunto de herramientas acordes al contexto. La indisciplina como un problema en sí mismo se ve agravado como resultado de los recursos equivocados para hacerle frente, además de la 
incapacidad y transigencia para atenderla con oportunidad se derivan otros más, y ésta se torna cada vez más complicada.

La disciplina escolar constituye una herramienta necesaria para dar cumplimiento a los compromisos del profesorado; desempeñarse como facilitador del aprendizaje y gestor de la convivencia en un grupo. Exige un marco de acción con referentes apropiados.

Sobre esta perspectiva teórica y en alusión a su fundamentación psicopedagógica al hacerla efectiva en clase, el estudio presentado hace un análisis de los vectores y recursos precisos que debe tener el profesorado para establecer de manera óptima la disciplina en clase teniendo como soporte su enfoque preventivo. Cabe remarcar de nuestro estudio, alguna limitación. Respecto a la muestra de 51 profesores; un $94.1 \%$ correspondieron al sexo femenino y $5.9 \%$ masculino como se ha indicado cosa que puede que haya tenido que ver con las respuestas encontradas.

Pese a las dificultades encontradas se puede afirmar que después del programa de formación sobre disciplina escolar se hallaron diferencias significativas en los conocimientos del profesorado, quienes cambiaron tanto sus conocimientos como sus actitudes.

\section{5.- REFERENCIAS BIBLIOGRÁFICAS}

Calvo, P., García A., y Marrero, G. (2005). La disciplina en el contexto escolar. Las Palmas de Gran Canaria: Universidad de las Palmas de Gran Canaria.

De la Mora, C. (2003). La disciplina en el aula. México: Aula Nueva.

Estrela, M.T. (2005). Autoridad y Disciplina en la Escuela. Sevilla: Trillas.

Fontana, D. (1994). El Control del comportamiento en el aula. Barcelona: Paidós.

Gotzens, C. (1997). La disciplina Escolar: Prevención e intervención en los problemas de comportamiento escolar. España:Ice- Horsori.

Imbernón, F. (1998). La formación permanente el profesorado. En Organización de Estados Iberoamericanos (Ed.), Una Educación con calidad y equidad (pp. 128-167). Barcelona. Biblioteca de Aula. pp.125-167.

Jones, V., y Jones, L. (1990). Classroom Management, Motivating and Managing Students. USA: Allyn and Bacon.

Lepage, P., Darling-Hammond, L. Akar, H. Gutierrez, C. Jenkins-Gunn, E., y Rosebrock, K. (2005). Classroom Management. En L. Darling-Hammond y J. Bransford (Eds.), Preparing teachers for a changing world, what teachers should learn and able to do (pp.327-357). San Francisco: Jossey-Bass.

Veiga, E., y Rodríguez, E. (2008). Desarrollo integral de la convivencia en el marco educativo: una nueva perspectiva del observatorio autonómico al observatorio escolar. Revista Galego-Portuguesa de Psicología e Educación, 16(12), 45-64.

Zamudio, R. (2009). Estudio exploratorio de los conocimientos el profesorado de educación básica para establecer la disciplina en el aula. Barcelona: Universidad Autónoma de Barcelona.

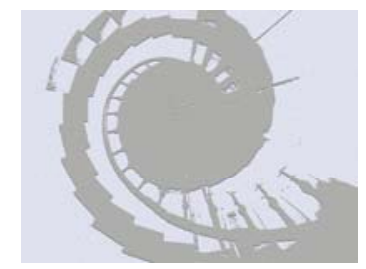

